\title{
1. Strategic innovation for sustainable growth: reviews of existing capabilities theories, and new propositions
}

Strategic innovation dynamically brings about strategic positioning through new products, services and business models, and is a dynamic view of strategy that enables a large corporation to maintain its competitiveness and establish sustainable growth. For these reasons, large corporations have to be innovators that can reinforce their existing positions (businesses) through incremental innovation, while at the same time constantly renew or destroy existing business through radical innovation.

From detailed reviews of existing capabilities theories (resource-based theory of the firm and dynamic capabilities, and so on), and further theories deeply related to the characteristics of corporate or organizational capabilities and field data on sustainable growth of global corporations, this chapter presents the concept of a "Capabilities Map" derived from existing research into the characteristics of dynamic capabilities responding to environmental conditions such as dynamic temporal shifts and factors of uncertainty.

\subsection{SUSTAINABLE GROWTH THROUGH RADICAL AND INCREMENTAL INNOVATION}

Here in the 21st century, changes in business circumstances surrounding large corporations are becoming more pronounced. Managers and business leaders face a wide range of challenges as businesses globalize and increasingly operate in emerging markets, as technologies innovate rapidly, networking permeates throughout societies and markets mature, as well as price wars and environmental problems. Obviously, a large corporation must continuously create new products, services and business models to maintain sustainable competitiveness and growth over the long term.

The creation of new business models that change existing rules and 
radically revamp conventional products and services trigger major transformations in the corporate strategy of large corporations. For example, new value chains and business ecosystems in the ICT industry that originated in the United States with the creation of a new music distribution system and smartphones with Apple's iPhone, iPod and iTunes music store caused major shakeups in both the music and mobile phone industries.

The collection of innovation research of recent years into radical innovation (e.g., Leifer et al., 2000), breakthrough innovation (e.g., O'Connor et al., 2008; Hargadon, 2003), discontinuous innovation (e.g., Kaplan et al., 2003) and disruptive innovation (Christensen, 1997) has offered both theoretical and practical pointers for transforming strategies in large corporations to advance technologies and create new markets. The important implications put forth by existing research suggest it is not enough to just be able to respond quickly to environmental changes - companies also need to acquire capabilities to develop business and create new environments (markets).

Thus, to swiftly respond to changing circumstances, companies have to continually polish their existing capabilities to fortify their main businesses. Incremental innovation (e.g., Ettlie, et al., 1984; Dewar and Dutton, 1986) through strengthening and utilization of company capabilities through regular, continuous and cumulative upgrade and improvement activities is important. At the same time, to drive business for radical innovation to create new environments (markets) (e.g., Henderson, 1993; McDermott and O'Connor, 2002), it's also necessary to seek out or create never-before-seen capabilities to drive business development. In these two innovation processes - incremental (exploitation) and radical (exploration) innovation - it's the former that entails strengthening and utilizing capabilities for a company's existing business (its main business) and pursuing greater operational efficiency. In contrast, it's the latter that involves a large corporation searching out or building new capabilities in pursuit of the creativity needed to pioneer new business models and the new businesses of the future. However, large corporations have to manage these two totally different innovation processes simultaneously and incorporate both of them into the nuclei of their corporate strategies.

The traditional large corporations of the past found great competitiveness in reinforcing and utilizing their path-dependent capabilities to incrementally innovate their existing products and profit by releasing newer versions of them. In contrast, radical or breakthrough innovations result in paradigm shifts accompanied by new markets and technologies that bring big increases in product functionality, radical changes to existing markets, the creation of new markets and substantial cost reductions (Leifer et al., 2000; O'Connor and Rice, 2001). In this way, as new breakthroughs, 
radical innovations are different in character to the incremental and pathdependent innovations of the large corporations of the past. To achieve radical innovation, large corporations need to seek out and create new capabilities that are different from their existing skills and know-how (e.g., Dewar and Dutton, 1986; Ettlie et al., 1984; Green et al., 1995).

However, corporations taking on radical innovation and various projects within themselves must face uncertainties and discontinuities in areas such as markets, technologies, organizations and resources. Hence, while it may be possible for some projects to overcome these hurdles, many projects lose momentum and fail (e.g., Leifer et al., 2000). To seek out and create new capabilities for radical innovation, large corporations must engage in management activities that are not the same as reinforcing and utilizing the capabilities they have nurtured through their incremental innovation histories (the business elements of strategy, organizations, resources, technologies processes and leadership) (e.g., Kodama, 2003; O'Reilly and Tushman, 2004; Vanhaverbeke and Peeters, 2005).

There is a dynamic relationship between the creation and utilization of these capabilities. Since strengthening and utilizing existing capabilities for incremental innovation means fostering and accumulating technical knowhow and personnel skills within companies, these capabilities can trigger the achievement of radical innovation through the searching and creation of new capabilities. Therefore, large corporations must understand their desired level (optimal level) of balance between creating and utilizing capabilities, and must intentionally manage them. Companies face new challenges regarding the creativity and efficiency of their capabilities, in other words "the combination of exploration and exploitation," to bring about radical innovation by seeking out and creating new capabilities, while at the same time maintaining their competitiveness through incremental innovation in their main businesses by strengthening and utilizing existing capabilities (March, 1991).

Thus, leaders and managers of large corporations need a perspective on seeking out and creating new capabilities to pioneer new business and create new markets while strengthening and utilizing capabilities to maintain their core businesses. Simultaneously executing and combining these two substantially different innovation processes entails the pursuit and pioneering of new and highly individualized strategic positions, and is thus a superior corporate strategy that also leads to the achievement of sustainable competitiveness and growth (e.g., Markides, 1999; Kodama, 2006).

In this book, the strategic activities or innovation processes that achieve a combination of radical and incremental innovation, or in other words combine the exploration and exploitation mentioned earlier, the author calls "strategic innovation." It's this strategic innovation that achieves 
sustainable growth in large corporations. Nevertheless, the questions remain - how do large corporations bring about strategic innovation, and what kind of strategic management in (and between) leading companies is needed to achieve sustainable growth? This book answers these holistic research questions from the perspective of academic research into strategic management and innovation.

\subsection{DYNAMIC CAPABILITIES AND STRATEGIC INNOVATION}

Markides (1997) defined strategic innovation as the dynamic creation of creative strategic positioning from new products, services, and business models, and emphasized that this framework was a dynamic view of strategy by which a company establishes sustained competitive excellence. To achieve this, companies must not adhere to existing positioning (existing business), but must always innovate in ways that destroy this positioning. Moreover, Govindarajan and Trimble (2005) defined it as realizing strategically innovative new business models (including new products and services). This strategic innovation refers to business innovation that transforms the established into new business and has a major impact on corporate performance, and is different in essence from incremental innovation.

Nevertheless, it goes without saying that incremental innovation drives existing business, and is a business activity crucial to making gains in the visible near term, but from there, companies must also keep an eye on initiatives to achieve radical innovation to secure sustainable growth into the future while investing their acquired short-term profits in new R\&D activities. In other words, companies have to strengthen their incremental innovation activities, while simultaneously directing themselves toward the challenge of radical innovation (e.g., Christensen, 1997; Goold and Cambell, 2002; Heller, 1999; Kodama, 2003, 2004; Tushman and O’Reilly, 1997; O'Reilly and Tushman, 2004). Thus, in this perspective, both incremental innovation and radical innovation must be simultaneously pursued in the strategic innovation implied by Markides (1997) and Govindarajan and Trimble (2005).

Based on a number of major studies, in particular those done on dynamic capabilities (Teece, 2007, 2014), this chapter clarifies the dynamic innovation processes of companies for establishing strategic innovation (incremental innovation and radical innovation) for sustainable growth. The characteristics of the capabilities that corporations use to handle such externalities as the fast environmental changes and uncertainties they face 
are described by the Capabilities Map concept (as the four capabilities domains).

\subsection{CAPABILITIES THEORY - DISCUSSIONS FOCUSING ON DYNAMIC CAPABILITIES}

The resource-based theories that focused on independent capabilities for companies and organizations (e.g., Penrose, 1959; Richardson, 1972; Wernerfelt, 1984; Rumelt, 1984; Barney, 1991) became strategic theory frameworks from the viewpoints of microeconomics and organizational economics. These resource-based theories and Porter's (1980) competition strategy theory enable a detailed analysis of strategic positioning and the relationship between competitive excellence and internal resources already owned by companies in slowly changing environments and industries. However, it is difficult to analyze how companies create new competitive excellence in rapidly changing high-tech industries in competitive environments such as the ICT and digital sectors.

In recent years, the theory of dynamic capabilities (DC hereinafter) (e.g., Teece et al., 1997; Teece, 2007, 2014) has been developed and refined, and has become a fundamental theory that clarifies the mechanisms for sustainable growth through corporate strategic innovation. Teece et al. (1997, p.516) define DC as the firm's ability to integrate, build, and reconfigure internal and external competences to address rapidly changing environments, Thus, they assert that DC reflect an organization's ability to achieve new and innovative forms of competitive advantage given its path dependencies and market positions (Leonard-Barton, 1992). Moreover, Teece (2014, p.332) also asserts that strong DC help enable an enterprise to profitably build and renew resources and assets that lie both within and beyond its boundaries, reconfiguring them as needed to innovate and respond to (or bring about) changes in the market and in the business environment more generally.

As core, micro level functions, DC can usefully be broken down into three primary clusters: (1) identification, development, co-development, and assessment of technological opportunities in relationship to customer needs (sensing); (2) mobilization of resources to address needs and opportunities, and to capture value by taking opportunities (seizing); and (3) continued renewal (transforming). Engagement in continuous or semicontinuous sensing, seizing, and transforming is essential if a company is to sustain itself as customers, competitors, and technologies change (Teece, 2007, 2014).

Regarding the area in which DC are applied, Teece et al. (1997) 
claimed that DC are important for sustainable company-level competitive advantage, especially in high-velocity markets. As well as that, strong DC enable an enterprise and its top management to develop assumptions about the evolution of consumer preferences, business problems and technology, validate and fine-tune them, and then act on them by realigning assets and activities to enable continuous innovation and change (Teece, 2014).

In this perspective, $\mathrm{DC}$ can be thought of as a dynamic business process that should be demonstrated in business environments that are changing rapidly, and/or in business environments that have high levels of uncertainty. It is especially important to demonstrate this DC in Domains I, II, III in the Capabilities Map in Figure 1.1 (business environments that are changing rapidly, and/or business environments that have high levels of uncertainty) discussed later.

In the dynamic environments of "hypercompetition" (D'Aveni, 1994) or "next-generation competition" (Teece, 2012a) gaining attention in recent years, the theoretical concept of DC has become crucial for companies to drive "ecosystem strategies" (Teece, 2014). Moreover, asset orchestration (Teece, 2007), a core function of DC, is reinforced by the organizational processes of (1) coordination/integration, (2) learning, and (3) reconfiguration (Teece et al., 1997). Affecting performance in the individual domains in the Capabilities Map (Figure 1.1) (discussed later), asset organization also has a profound relationship with the core theoretical "capabilities congruence" framework of this book (discussed in Chapter 3).

Teece $(2007,2014)$ clearly distinguishes these DC from "ordinary capabilities" (OC hereinafter). Teece (2014, p.330) states:

Ordinary capabilities have also been called static (Collis, 1994), zero-level (Winter, 2003), first order (Danneels, 2002), and substantive (Zahra, Sapienza, and Davidsson, 2006). The zero-, first-, and second- typology is used by Easterby-Smith and Prieto (2008) and Schilke (2014). The more common usage seems to be equating first-order with ordinary.

These OC generally fall into three categories: administration, operations, and governance. In describing specific details of corporate activity, OC enable a firm to perform activities on an ongoing basis using more or less the same techniques on the same scale to support existing products and services for the same customer population. Such capabilities can be said to be ordinary in the sense of maintaining the status quo (that is, not out of the ordinary; Winter, 2003) (Helfat and Winter, 2011).

Nevertheless, in the pursuit of efficiency in terms of a company's best practices and "doing things right," OC are not to be underestimated they are often fundamental and can support competitive advantage for 
decade-long periods (Teece, 2014). In other words, in relatively stable environments where environmental change is gradual and there are low levels of uncertainty, OC function usefully in business, but don't secure corporate sustainable growth over the long term. However, in traditional large corporations engaging in wide-ranging business activities, there are always some business domains in which OC must be demonstrated - these OC are critical for business in slow or relatively stable environments of low uncertainty, and are particularly important in Domain IV in Figure 1.1 (low uncertainty, slow environmental change), as discussed later.

Therefore, companies apply OC and systematically and analytically formulate and implement strategies under relatively stable or slow-moving conditions with little business uncertainty. "Learning before doing" (Pisano, 1994), that is, formulating and implementing detailed strategy planning and policies, is a key element of $\mathrm{OC}$ in market structures with clear corporate boundaries and where there is a good grasp of the players in value chains.

In contrast, DC have been reinterpreted by a number of researchers, and among those, Eisenhardt and Martin (2000) have been quoted in many papers (Web of Science: 2451, as of 2015). They express DC as "The firm's processes that use resources - specifically the processes to integrate, reconfigure, gain and release resources - to match and even create market change. Dynamic capabilities thus are the organizational and strategic routines by which firms achieve new resource configurations as markets emerge, collide, split, evolve, and die" (Eisenhardt and Martin, 2000, p.1107) and recursively extract the concept of corporate DC required in both slow and fast-moving business environments. They emphasize "learning by doing" with simple rules focusing on results rather than prior training and implementation processes, especially in fast-moving environments, where uncertainty rises and an industry's corporate borders become vague (Eisenhardt and Sull, 2001). However, Eisenhardt and Martin (2000) claimed that DC are inherently unsuited to creating sustainable advantage and that they are likely to break down in high-velocity markets.

In contrast to this assertion by Eisenhardt and Martin (2000) that "dynamic capabilities would break down in high-velocity environments because of the instability of the simple rules (basically, semi-improvised managerial actions)," Teece (2014, p.339) states that:

In high-velocity environments, the business enterprise may well be particularly reliant on the sensing and seizing instincts and actions of the CEO and the top management team. To the extent that this is so, the capabilities will, of course suffer from a degree of instability because their longevity depends logically on the tenure of entrepreneurs/managers/leaders. 
Viewed from the perspective of a practitioner, Teece's comments (2014, p.339) can be seen as more rational reasoning that captures the real business environment. Moreover, considering the author's experience of having worked on a number of product planning projects to date, DC are also the quick-wittedness required by the dynamic spiral thinking and action (simultaneously both deliberate and emergent) of regular trial and error of project leaders and team members on the front line of new processes for building new business, and are not just the attributes of top management in high-speed, rapidly changing environments. The author doesn't negate the idea of simple rules, although it should be one standard of judgment that practitioners use in complex and dynamic business processes in response to certain situations (not all situations).

In contrast to the discussions of Eisenhardt and Martin (2000), Teece (2014, p.332) asserts that:

Eisenhardt and Martin's (2000) article misinterpreted (or reframed) the DC framework by claiming that all capabilities, including DC, can ultimately be characterized by best practice and hence imitated. In essence, Eisenhardt and Martin conflated two concepts that benefit from being analytically separated, namely OC and DC. OC and DC are quite distinct, both analytically and in practice.

Hence, it appears different researchers have different positions on the interpretation of DC.

Nevertheless, through some other researchers, this interest in strategy theory has evolved toward a dynamic structure that reflects current corporate activity. For example, O'Connor (2008) respects the DC theory of Eisenhardt and Martin (2000), and mentions that a large number of major innovations (MI) (included in the concept of strategic innovation in this book), including radical innovations, developed gradually from slow (or very slow) market environments, and were implemented over a period of several years to several decades. Thus, the concept of DC is described as a theory that can be evaluated and applied around the axes of both market speed and business uncertainty (including risk), and is characterized by strategic innovation.

In addition, O'Connor (2008) used the term "MI dynamic capability" for the capability that promotes the "exploration" process (March, 1991) and realizes strategic innovation under conditions of uncertainty and high risk. MI dynamic capability differs from the capability theory (e.g., King and Tucci, 2002; Nelson and Winter, 1982; Winter, 2000) that emphasizes the evolution of the original "exploitation" (March, 1991) activity process. MI dynamic capability responds to highly uncertain situations, regardless of the speed of market movement, and embraces the concept of DC in the 
high-speed (and highly uncertain) markets mentioned by Eisenhardt and Martin (2000).

Realistically speaking (including the author's practical experiences), many strategic innovations are established through discovery or invention in slow - and very slow-moving basic scientific research and technological development environments. Later, the developed core technologies and provisional business models based on discovered or invented ideas are adopted and exploited in products and services through improvisation and trial-and-error processes (including the weeding-out process) involving trial manufacture, experiment, and incubation. Product and service markets are gradually established. Then, new products and services anticipated or forecast for growth spurn competitive markets involving other companies (just when other companies enter the market depends on individual businesses). These market environments become fast-moving, and companies accelerate their investment in necessary resources.

O'Connor and DeMartino (2006) undertook long-term observation and analysis of radical innovation in major US corporations, and identified the importance of three-phase management (discovery, incubation, and acceleration) as a radical innovation (part of strategic innovation) development framework. They then named the ability to implement these processes the "breakthrough innovation capability," and suggested that building this capability into a company is a key managerial approach that can lead to successful radical innovation (O'Connor et al., 2008).

This three-phase management (discovery, incubation, and acceleration) is used in projects in large corporations (and venture enterprises) to develop various new products, services, and businesses. Different practitioner (and organizations such as project teams) capabilities are required in the individual business processes in each of the three phases, depending on the degree of business uncertainty and environmental changes being faced. As mentioned, DC robustly function in response to these externalities (uncertainty and environmental change), and are also a framework for demonstrating difficult-to-imitate competitiveness. Therefore, the three phases of Breakthrough Innovation Capability (O'Connor et al., 2008) (discovery, incubation, and acceleration) or MI dynamic capability (major innovation) (O'Connor, 2008) can be described as the three DC functions (sensing, seizing, transforming) which can be applied in high-speed and uncertain environments, and even in its theoretical concepts, DC can also be said to include the frameworks for Breakthrough Innovation Capability (discovery, incubation, and acceleration) and MI dynamic capability (major innovation).

Previous research, such as Teece's DC framework and MI innovation capability, is positioned around the two axes of uncertainty and change and led to the situation illustrated in the Capability Map in Figure 1.1, 


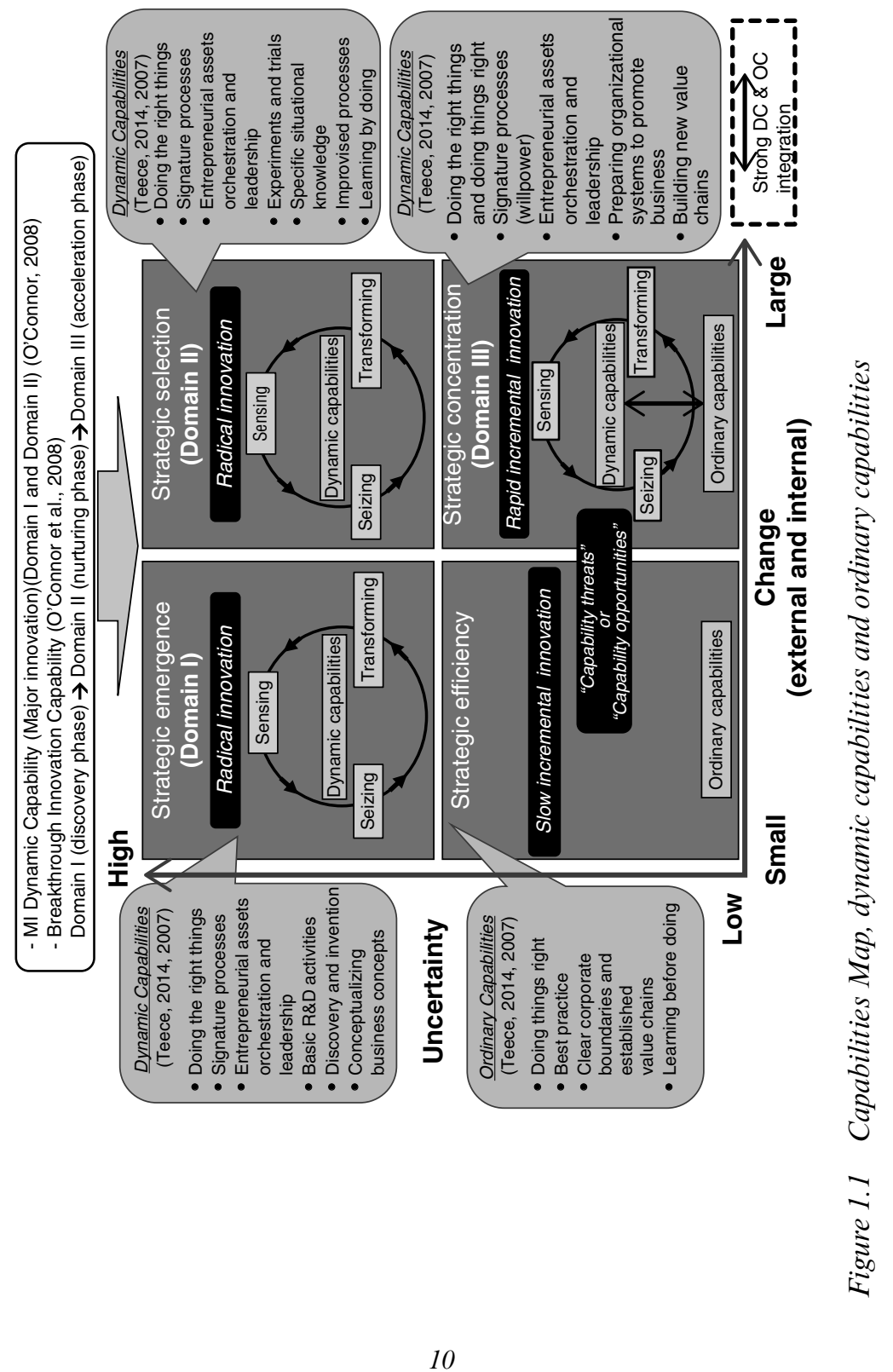


which shows the relationship between those previous researches and the three development phases of O'Connor and DeMartino (2006) mentioned above. As mentioned, three-phase management (discovery, incubation, and acceleration) entails moving in order through Domain I, Domain II and Domain III, from business conception or invention to practical realization, which are fields of business in which DC are demonstrated (and OC in Domain III as discussed later). In contrast, it is OC that function in pursuit of best practices (Teece, 2007, 2014) in slow and stable environments with low uncertainty and change (Domain IV). Here, strategic uncertainty beyond the four elements of markets, technology, organization and resources mentioned by Leifer et al. (2000) also exists, and change is not limited to the external elements of market or industrial technology speeds, but also corresponds to the internal elements of a company's own strategy - organization (organizational revamping), technology, operation and leadership (described in detail in Chapter 3). The following describes the characteristics of capabilities in each Domain.

\subsection{THE CAPABILITIES MAP - THEORETICAL FRAMEWORKS}

Based in the theoretical concepts of the exiting research described above, this book analyses the capabilities required by the various organizations in large corporations (organization charged with R\&D and new business development, project teams, and existing traditional organizations, and so on) in the various business contexts that they regularly face, and provides a new theoretical framework.

\subsubsection{DC in Domain I}

Slow or very slow environmental change with a highly uncertain domain (Domain I) observed at the initial stage of strategic innovation is the technology creation stage arising from new ideas, business concepts, discoveries, and invention, and corresponds to the "discovery phase" described by O'Connor and DeMartino (2006). In this domain, the exploration process advances through the MI dynamic (or breakthrough innovation) capability mentioned above. The role of sensing is important in DC. In R\&D organizations in major corporations (laboratories, development centers, and so on) $\mathrm{R} \& \mathrm{D}$ organizations set down and execute mid- to long-term plans either continuously or semi-continuously, which entails seizing and transforming processes while searching out and detecting latent new markets through sensing to achieve radical innovation. 
The basic research and creation of ideas that are the source of new strategic innovation require (depending on the field) a longer period of time as the ratio of scientific elements and the degree of technological difficulty rises. In this domain, achievements are often the result of the creative thinking and actions of middle managers and staff in company $\mathrm{R} \& \mathrm{D}$ departments and business development divisions, but there is also substantial commitment and strategic contribution made by top management and upper level managers based on the policies of "doing the right things"(Teece, 2014). Moreover, there are important "signature processes" (Bruch and Ghoshal, 2004) in large traditional (leading) corporations that are difficult for other companies to copy, which are processes that raise the quality of R\&D. The author calls this domain the "strategic emergence" domain.

In the asset orchestration process in this domain, practitioners have to pursue reconfiguration and transformation through coordination and integration of various intangible assets as well as engage in learning as hypothetical testing tailored to the their $\mathrm{R} \& \mathrm{D}$ aims. There are a variety of asset orchestration patterns, and there are still many cases in many traditional large companies under conventional hierarchical systems of closed innovation centered on internal laboratories and development divisions (Japanese manufacturing is a typical example). To develop incremental innovation or sustain innovation through accumulated path-dependent knowledge (Christensen, 1997), closed innovation is still an important process. Closed innovation plays a critical role in traditional high-tech fields such as the heavy electrical, nuclear power generation, aviation, vehicle equipment, machine tool, medical and semiconductor machinery industries. In contrast, in industries in which technologies are rapidly advancing such as ICT, the best technical achievements and know-how are becoming increasingly spread out across the globe. In these fast-moving environments, open innovation is adopted (Chesbrough, 2003), partial core intangible assets are incorporated from externalities, and hence processes to merge and integrate intangible assets both within and from the outside of companies become critical. Here, of particular importance are the processes of coordination and integration in asset orchestration of various resources performed by top and middle managers in an entrepreneurial fashion.

In Domain I, companies must consider what business models they are seeking. Should a company adopt a vertical integration model with the aim of finally bringing about completed parts, products and services, and so on, or adopt horizontal integration to focus on the company's particular area of expertise? Should a company reinforce its technologies while searching out strategic alliances (strong or weak ties) with other companies? Should 
a company build new value chains through coordination and integration of intangible assets, which means using the strengths of the company and other companies forged though strategic collaboration across different types of business. Thus, in allowing for expanded diversification of asset orchestration, practitioners have to concentrate on learning through trial and error, experiments and trial activities.

In strategic emergence in Domain I, companies have to hypothetically test their corporate boundaries in response to strategic objectives or business environments and make attempts at reconfiguration/transformation of diverse entrepreneurial asset orchestration through processes of trial and error. If it's advantageous to develop or manufacture in-house, then it's better to configure a vertical value chain model with a focus on creativity (Kodama, 2009a). In contrast, if another company has achieved more with its developments than those in-house, there are many cases in which a company should abandon its development efforts, and focus on efficiency not only through strategic outsourcing but also through strategic alliances, joint developments and M\&A to access and acquire external intangible assets. The important factor of this asset orchestration process is the "co-specialization" described in Chapter 3. Co-specialization is the way the levels of synergies of technologies and so forth are raised in business, and the process of co-specialized assets orchestration is an important factor in raising a company's "dynamic internal and external congruence in capabilities."

\subsubsection{DC in Domain II}

Next, with rapid changing of in-house (or occasionally external) human resources and maintenance and upgrading of organizations oriented to business incubation, core technologies and business concepts migrate from the slow-moving environment of Domain I to a dramatically transforming Domain II environment that is full of rapid change and uncertainty. In this domain, the exploration processes arising from DC (MI dynamic or breakthrough innovation) described by O'Connor (2008) are promoted. This domain corresponds to the incubation phase of hypothetical setups, experiments, and assessments mentioned by O'Connor and DeMartino (2006). Learning through trials and experiments also leads to less risk and uncertainty with markets and technologies and greater probability of success for incubations aimed at realizing strategic innovation (O'Connor et al., 2008). Then, top and middle management can make decisions to select and bring to market rigorously tested products, service, and business models.

In Domain II (the incubation phase), the role of seizing is important in 
commercial development divisions on the business side to achieve radical innovation. Commercial development divisions "sense" matches between the market and technical innovations while seizing and transforming for radical innovation as the commercial development of new processes, new technologies and new businesses. Thus, practitioners pursue entrepreneurial strategies, demonstrate commitment and make strategic contributions based on the basic policy of "doing the right things." Moreover, the quality of the signature processes unique to a company that were required in Domain I are more strongly reflected in this domain. This is because there exist serious hurdles called the "valley of death" (Kodama, 2011) between R\&D and commercialization of its achievements, and the ability to surmount these hurdles largely comes down to these rarified signature processes unique to companies.

O'Connor et al. (2008) confine this incubation domain to trials and assessment models, but in many cases current business activities go beyond trials in uncertain and dramatically changing, fast-moving environments to the launch of commercial businesses, where companies might boldly undertake risks. In this domain, there are numerous cases of excessive trust and commitment of leaders and managers leads toward strategic activity based on the creation of business through trial and error, although whether newly developed ideas and prototypes are capable of becoming new business models and value chains might still remain to be seen. These enterprises are typical of the new online business world where products are both trialed and launched in dramatically changing, risky and uncertain domains. The key here is being able to select and implement promising, valuable businesses, which is why the author calls this domain the "strategic selection" domain.

In this domain, the asset orchestration process entails selection and narrowing down and refining the diverse intangible and tangible assets trialed and experimented on in the strategic emergence domain. Following, the level of completeness of asset orchestration as products, services and business models is raised through the processes of (1) coordination/integration, (2) learning, and (3) reconfiguration. Hence, depending on circumstances, there are cases where a corporation has to rethink its corporate boundaries (both vertical and horizontal) or its relationships such as partnerships with other companies, and realign or reconfigure its assets.

\subsubsection{DC and OC in Domain III}

New businesses (including new products and services) chosen through strategic selection in Domain II that have future prospects and somewhat reduced uncertainty shift to the more certain Domain III, although 
external (environmental) and internal change continues. In Domain III, the strategic innovation incubated (or partially commercialized) in Domain II enters a growth trajectory which corresponds to the "acceleration phase" discussed by O'Connor and DeMartino (2006). According to O'Connor et al. (2008), this is where the exploitation process is promoted by Breakthrough Innovation Capability. Hence, this is the domain where building and optimization of processes and value chains for selected new businesses is achieved.

These new business functions are wholly or partially transferred to the appropriate business divisions to accelerate commercialization (or new business divisions are established, or made independent as external ventures), and further resources are intensively invested through "doing the right things" and the strategic commitment of top and middle management. Hence, the author calls this domain the "strategic concentration" domain. In the past, a large number of product and service development projects for major corporations (e.g., Kodama, 2005; 2007d) invested management resources through asset orchestration in commercialization through this shift from strategic selection to strategic concentration.

In Domain III, a domain in which the pace of environmental change is fast and competition with other companies is fierce, "transforming" on the business side plays an important role. With the passing of time, newly developed products and businesses will burst into the competitive environments with other companies at the stage of shifting into Domain III. Nevertheless, the shift to a competitive environment depends on the industry or the features of a product, and in this sense the birth of a competitive market means that uncertainty lowers in the environment, in other words the market. In contrast, divisions such as product planning and technical development positioned on the upstream of the value chain at the business side (sales HQ and sales divisions, and so on), also function to sense and detect changes in the market that have been newly brought about, and establish robust value chains through seizing and transforming for upgrades and improvements by rapidly and incrementally innovating new products and services (sustainably advancing the technologies) that have been successfully commercialized. For this reason, practitioners pursue entrepreneurial strategies here (including deliberate and emergent strategies), and demonstrate commitment and strategic contribution based on the basic policy of "doing the right things."

Moreover, in Domain III, there is a significant dependence on the "willpower" (Bruch and Ghoshal, 2004) of the unique signature processes of a company to win out over the competition. Willpower is the energy and concentration of the thinking and action that accompany a sense of purpose. Energy means vigor, and concentration directs this vigor toward 
a particular goal. Practitioners paint a clear picture of their intended strategy in their minds, with the most important factor being that they consciously dedicate themselves to planning to bring their intended strategy into being in the midst of stiff competition. Moreover, in this domain, it's the willpower in the unique and highly rarefied signature processes of a company that carries much of the burden. Just as Teece (2014, p.341) argues, a strategy can be defined as "a coherent set of analyses, concepts, policies, arguments, and actions that respond to a high-stakes challenge" (Rumelt, 2011, p.6). The best strategies require (1) a diagnosis, (2) a guiding policy, and (3) coherent action brought about by the unique signature processes of a company based on willpower (Rumelt, 2011). Currently, the smartphone industry is in this Domain III stage.

In Domain III, the completion level of products and services is raised through upgrades and improvements for rapid incremental innovation following commercialization, through the processes of asset orchestration promoted and concentrated to complete value chains. Through trial and error, although the changes in factors of the asset orchestration process are lower than the strategic selection in Domain II, the most important issue is overall optimization (external and internal congruence in capabilities) of the corporate system, as described in Chapter 3.

However, in Domain III, to get new products, services and businesses off the ground and win out over the competition, robust value chains must be configured. As mentioned, organization supervisors and staff in product planning and technical development divisions on the business side upstream in the value chain must demonstrate strong DC, however in contrast, staff and leaders in routine divisions on the downstream in the value chain (sales, technical management, procurement, manufacturing and after support etc.) need thoroughly reinforced operations management enabled through strong OC. These downstream organizations invest in current products (and their successor upgraded and improved versions), and require strong OC to win out amid stiff competition and turn a profit. Thus, the characteristics of the capabilities required to promote business in Domain III are not the same as those in Domains I and II, and have a particularly strong focus on integration of DC and OC (see Figure 1.1).

\subsubsection{OC in Domain IV}

A great deal of existing business is positioned in Domain IV, in slow-moving market environments with low uncertainty and a low rate of change. Here, incremental innovation is promoted to systematically enhancing business efficiency through the exploitation process, which entails activities to 
improve existing business using mainstream organizations that demonstrate inherent OC (Teece, 2007, 2014).

In Domain IV, the weight on DC diminishes, and the focus shifts to the demonstration of best practices through OC. Existing traditional organizations (business divisions, and so on) detect slow changes in existing markets, and execute existing operations at formal organizations through strict top-down centralized leadership (Kodama, 2004), path-dependent planning in business divisions and well thought-out deliberate strategies. In Domain IV, high performance must be brought about by evolving routines through higher-order learning to generate short-term profits in response to internal and external changes, and to drive slow incremental innovation through strengthened OC (King and Tucci, 2002; Benner and Tushman, 2003; Winter, 2000; Amburgey et al., 1993; Nelson and Winter, 1982). Promoting this Domain IV process management accelerates an organization's speed of response to achieve incremental innovation (Benner and Tushman, 2003). However, as discussed later in Chapter 2, there is always a danger that product lineups in this Domain could be threatened by emergent technical innovations. The author calls this domain the "strategic efficiency" domain.

Most of the businesses in Domain IV (products and services) are survivors from the competitive environment in Domain III that have moved into Domain IV, a domain that entails shifting from old to new business over long periods of time (Markides, 2001). In other words, existing business in the strategic efficiency Domain IV might be subjected to this old-new business conversion into a new strategic concentration (Domain III) business as a path newly created through strategic innovation (Domain I $\rightarrow$ Domain II $\rightarrow$ Domain III). Markides' (2001) discussion of simultaneously managing existing and new strategic positions implies the combination of Domains IV and III respectively, and that the shift from an old position to a new one implies (all) existing business in Domain IV can shift to accelerate and grow as new business in Domain III.

As above, in describing the dynamics of the shifts between domains in the Capabilities Map, of particular importance are the strategic actions in Domains III and IV that aim for sustainable growth through ongoing corporate strategic innovation. According to the "Capabilities Lifecycles" framework of Helfat and Peteraf (2003) (described in section 2.3 in Chapter 2), to achieve further strategic innovation, companies have to uncover "capability opportunities" and sometimes deal with "capability threats" by driving new DC in Domains III and IV, which hinges on the shift to Domain I (see Figure 1.1). In short, through dynamic interaction with environmental changes, leading major corporations engage in spiraling strategic activities between these four domains to achieve strategic 
innovations (Domain I $\rightarrow$ Domain II $\rightarrow$ Domain III $\rightarrow$ Domain IV $\rightarrow$ Domain I and/or Domain III $\rightarrow$ Domain I $\rightarrow$ and so on) (discussed in detail in Chapter 2).

\subsection{CHAPTER SUMMARY}

This chapter presents the concept of a Capabilities Map derived from existing research into the characteristics of DC responding to environmental conditions such as dynamic temporal shifts and factors of uncertainty (see the four capability domains in Figure 1.1).

However, increasingly important is research from a strategic and organizational perspective on capabilities needed to achieve sustainable growth through continuous strategic innovation - the systematic incremental and radical innovation in large corporations which does not depend on black box capabilities or the capabilities of certain practitioners. Compared to startups and venture companies, large corporations have greater wherewithal for new routines and learning through experiment or trial and error (Floyd and Wooldridge, 1999; Kogut and Zander, 1992). However, not much theoretical or empirical research into continuously and systematically bringing about strategic innovation (incremental and radical innovation) to achieve sustainable growth in large corporations exists. For this reason, research must be promoted from the perspective of corporate and management systems that achieve sustainable growth.

Following, Chapter 2 discusses capability factors needed for sustainable growth through the continuous execution of radical and incremental innovation (strategic innovation). 